\title{
Evaluation ofIL-6 and TGF- $\beta 1$ level in serum and saliva of patients with systemic sclerosis
}

\author{
Dr. Muhassad H. Al-mudhaffar ${ }^{1}$, Dr. Taghreed F. Zaidan ${ }^{1}$, Dr. FaiqI. Gorial ${ }^{2}$ \\ ${ }^{I}$ Department of oral diagnosis, Collage of Dentistry,Bagdad university/Iraq \\ ${ }^{2}$ Department of medicine, Collage of Medicine, Bagdad university/Iraq
}

\begin{abstract}
:
Introduction and purpose: Systemic sclerosis (SSc) is multi organs disorder with hyper reactive immune system reflected by excessive skin sclerosis and affected organs. Cytokines were release as basic procedures in SSc pathogenesis as they are convolute in $T$ and $B$ cell activation leading to inflammation, auto antibodies, and microvascular injury this entire event leading to fibrosis. This study is designed to investigate the changes of IL6 and Transforming growth factor beta 1 (TGF- $\beta 1)$ in the whole saliva and serum of patient with SSc.

Study design: forty female systemic sclerosis patient diagnosed previously by Rheumatologist in Bagdad teaching hospital in Bagdad cityfrom 2015-2016, serum and saliva werecollect, IL-6 and TGF-B1were investigate and statistical data compared with control subjects.

Method: The quantitative determination of TGF- $\beta 1$ was used from Elabscience Company, quantitative determination of human TGF- $\beta 1$ concentrations in serum, plasma and other biological fluids by ELISA technique.

The quantitative determination of IL-6was used from Elabscience Company that it was determined of human IL6 concentrations in serum, plasma and other biological fluids by ELISA technique.

Results: The present study showed that the median and mean rank level of serum IL-6 in SSc patients (18.27 and 55.93) was highly significantly increased $(P<0.001)$ as well as the mean level ofsalivary IL-6 in SSC patients $(7.084 \pm 1.984)$ was highly significantly increased $(P<0.001)$ than that the control subjects. The median and mean rank level of serum and salivary TGF- $\beta 1$ in SSc patient was highly significantly increased $(P<0.001)$ than control subject.

Conclusion: IL-6 and TGF- B1play a major role in SSc pathogenesis therefore both biomarker were investigate in serum and saliva ofSSc patients, saliva is non-invasive and accurate in detection ofthese markers.
\end{abstract}

Keywords: SSc, IL-6, TGF- $\beta 1$.

\section{Introduction}

Systemic sclerosis (SSc) is a chronic autoimmune illness, which is considerby presence of three major manifestations [1]:

- Fibrotic changesof in the skin and systemic organs,

- Non-inflammatory vasculopathy of small blood vessels, and

- Particular autoantibodies

Relatively, it is a rare disease with affecting women more frequently than men $(3: 1)$. The age of onset distinctively from 30-50 years, with a prevalence in the United States general population of $0.01-0.03 \%$ and worldwide distribution [2]. Systemic sclerosis was classify into two major types basing on the degree of clinically affected skin. theSSc divisions have been identified: the first one is scleroderma restricted to cutaneous only(lcSSc) and diffuse cutaneous scleroderma (dcSSc).DcSScis a comparatively short prodromal phase;considered by constitutional symptoms, arthralgias, puffiness of the fingers, and the new onset of Raynaud's phenomenon. The diffuse cutaneous form is characterize by rapid progression of skin changes from the fingers or feet to proximal involvement of theextremities extending above the knee or elbow or to areas of the trunk.In early stage of the disease, the skin inclines to be inflame edematous (nonpitting) and erythematous with varying areas of hyper-or hypopigmentation (vitiligo-like), skin and soft-tissue tightness, intense pruritus, and pain typically follow the initial phase of acute inflammation. The articular or periarticular inflammation or fibrosis is suggested by the detection of friction rubs (crepitus). Flexion contractures of joints (especially of the fingers, wrists, and elbows) can progress with significant associated functional impairment [3].

\section{Clinical presentation:}

Skin features:

The following skin features [4]: 
1. Non-pitting oedema is a common early sign of swelling fingers and toes that digits may appeare sausagelike; finger movement may be restricted.

2. Skin thickening - this may be restricted joint movement or cause joint contractures; this is known as sclerodactyle if associated with the fingers leading to decrease movements ofhand due toSwelling and sclerotic changesand called 'prayer sign'.

3. Digit ulcer- pitting or ulcers on tips of finger may have also loss of bulk from finger pads.

4. Raynaud's phenomenon- This is the mutual symptom and it is find at some point in $90 \%$ of patients. Raynaud's phenomenon with Puffy fingers is believed to be a mainmark of likely SSc.

5. Calcinosis - lumps or nodules of chalky material which may be disrupted through the skin

6. Face and mouth:

- Facial skin tightening.

- Decreased in mouth opening (microstomia) - can make dental hygiene difficult.

7. Telangiectasia.

8. Dry or itchy skin; skin areas that affected complain of hair loss.

\section{Task of cytokines in SSc pathogenesis:}

Systemic sclerosis (SSc) is multi organs disorder with hyper reactive immune system reflected by excessive skin sclerosis and affected organs. Cytokine were release as basic procedures in SSc pathogenesis as they are convolute in $\mathrm{T}$ and $\mathrm{B}$ cell activation leading to inflammation, autoantibodies, and microvascular injury this entire event leading to fibrosis [5].

\section{Interlukin-6 (IL-6):}

Interleukin-6 is a cytokine with pleiotropic behavior with dramatic role in acute-phase reactions and inflammation, as well as in adaptive immune reactions. Macrophages, lymphocytes and hepatocytes some of cells which producing IL-6, and responsible for regulator of the acute phase reaction in the liver, prompting the "C-reactive protein production (CRP), serum amyloid, fibrinogen and other mediators"[6].Several studies focusing on the pathogenesis of SSc, suggested that this cytokine have been dramatic role in stimulating fibrosis by increasing inflammation due to higher levels of serum IL-6 as well as in other biological fluid such as bronchoalveolar lavage fluid also can be notice in skin of SSc patients [7,8].IL-6 is a cytokine have proinflammatory role especially in autoimmune disorders, it is believable that these immune complexes could be contributed to disease development. In systemic sclerosis were anti-IL-6 raised, equivalent with IL-6 level. They proposed that the anti-IL- 6 operated as a carrier, neutralized IL-6 in circulation and prevent sending of the cytokine to target cells and tissues [9]. The level of IL-6 were amplified fourfold in dermal fibroblasts of systemic sclerosis when compared with fibroblasts of healthy controls and pulmonary B cells of SSc patient inducing IL-6 secretion from pulmonary fibroblast $[10,11]$.

\section{Transforming growth factor beta (TGF-B):}

Transforming growth factor beta (TGF- $\beta$ ) is a cytokine with multiple responsibilitythat adjusted the "proliferation, differentiation of cell, and production of extracellular matrix (ECM)"[12].

The most strongprofibrotic cytokine is Transforming growth factor beta because of it is thought to show a main role in SSc pathogenesis [13].TGF- $\beta 1$ is important for "wound healing, stimulates matrix molecule deposition", and it is associated in the fibrotic disorders pathogenesis including SSc. Because prior studies demonstrated that Sema-7a promotes TGF- $\beta 1$ induced injury and fibrosis, and that TGF- $\beta 1$ induces the accumulation of fibrocytes[14].

\section{Materials and methods}

This study was carried out during the period from the middle of November 2015 till the end of March 2016 in Baghdad city, the sample of this study was divided into two groups:

1- Forty systemic sclerosis patients: Those patients were treat at Rheumatology department in Bagdad teaching hospital in Baghdad city.

2- Forty healthy control subjects, age matched with no signs and symptoms of any systemic diseases.

All patients were diagnosed by a Rheumatology specialist as systemic sclerosis patients depending on the criteria of the ACR, $1982 \& 2013$. All the subjects answered a written questionnaire regarding their name, age, gender, occupation, dental and medical histories, regularity of tooth-brushing, feeling of dry mouth and bad breath, and any oral, dental and systemic diseases.Complete medical history with clinical and physical examinations for each individual included in this study was perform by Rheumatologist.

\section{Saliva sample:}


The whole resting (Unstimulated) saliva was gathered under inactive circumstances ranged from 8.011.0 a.m. Any oral hygienic procedureswere avoid and patients were ask to clean their mouth with water, create saliva in their oral cavity and spit into a test tube[15].

Saliva was centrifuge at "3000 rpm" for 10 minutes. The resulting supernatant from centrifuged saliva was stored at $-20^{\circ} \mathrm{C}$ in polyethylene tubes until analyzed.

\section{Serum sample:}

About $5 \mathrm{ml}$ of venous blood samples were permit to clot at room temperature for 2 hours from each subject. The samplewas centrifuge for " 15 minutes at $1000 \times \mathrm{g}$ ". Blood collection tubes should be disposable, non-pyrogenic, and non-endotoxin The quantitative determination of IL-6 Kitwas use from Elabscience Company that it was detect of human IL-6 concentrations in serum, plasma and other biological fluids by ELISA technique. The quantitative determination of TGF- $\beta 1$ Kitwas use from Elabscience Company that it was detect of human TGF- $\beta 1$ concentrations in serum, plasma and other biological fluidsby ELISA technique.

\section{Statistical analysis}

The documents data were transform into a computerized record structure. A professional statistical guidance was sight for statistical analysis were computer aided by using "SPSS version 21.0 Statistical Package for Social Sciences". The statistical analysis includes:

- Descriptive statistic: The quantitative result was tested by Kolmogorov-Smirnov Z test to determine whether these data was parametric or non-parametric. The normally distributed data (parametric data) was suitably described by "mean, standard deviation (SD)" while non-parametric data was suitably described by "median and mean Rank"

- Inferential statistic: These were used in order to accept or reject the statistical hypothesis.

$>$ Independent sample t-test was used to test statistical differences between two parametric groups.

$>$ Mann-Whitney test was used to test statistical differences between two non-parametric groups

\section{Serum and salivary IL-6 parameters:}

\section{Results}

The present study showed that the median and mean rank level of serum IL-6 in SSc patient (18.27 and 55.93) was highly significantly increased $(\mathrm{P}<0.001)$ using Mann-Whitney $U$ test than that control group $(7.35$ and 25.08).

The mean level of level of salivary IL-6 in SSc patient $(7.08 \pm 1.98)$ was highly significantly increased $(\mathrm{P}<0.001)$ using t-test than that control group $(5.96 \pm 2.71)$ as shown in table $(1,2)$.

Table (1):-The median and mean rank values of different Serum IL-6 in both SSc patients and control subjects.

\begin{tabular}{|l|l|l|l|l|}
\hline & Groups & Median & Mean Rank & P value \\
\hline \multirow{2}{*}{ Serum IL6 pg/ml } & patients & 18.27 & 55.93 & $<0.001$ \\
\cline { 2 - 4 } & Control & 7.35 & 25.08 & \\
\hline
\end{tabular}

Table (2):-The mean values of different Salivary IL-6 in both SSc patients and control subjects.

\begin{tabular}{|l|l|l|l|l|l|l|}
\hline & \multicolumn{2}{|l|}{ Patient (N0) } & \multicolumn{2}{l|}{ Control (N=40) } & \multicolumn{2}{|l|}{} \\
\cline { 2 - 7 } & mean & SD & mean & SD & t-test & P-value \\
\hline Saliva IL-6pg/ml & 7.08 & 1.98 & 5.96 & 2.16 & 2.71 & 0.000 \\
\hline
\end{tabular}

\section{Serum and salivary TGF- $\square$ parameters:}

The median and mean rank level of serum TGF- $\beta 1$ in SSc patient (135.4 and 56.85) was highly significantly increased $(\mathrm{P}<0.001)$ using Mann-Whitney $\mathrm{U}$ test than that in the control group (34.76 and 24.15). While concerning the salivary TGF- $\beta 1$ the median and mean rank level in SSc patients equal to (41.98 and $55.70)$ was highly significantly increased $(\mathrm{P}<0.001)$ using Mann-Whitney $U$ test than the control group $(22.6$ and 35.30$)$ as a shown in table $(3,4)$.

Table (3):-The median and mean rank values of different Serum TGF- $\beta 1$ in both SSc patients and control subjects.

\begin{tabular}{|c|c|c|c|c|}
\hline & Groups & Median & Mean Rank & $P$ value \\
\hline \multirow{2}{*}{$\begin{array}{ll}\text { Serum } & \text { TGF- } \\
\square \text { lpg/ml } & \\
\end{array}$} & patients & 135.4 & 56.85 & \multirow[t]{2}{*}{$<0.001$} \\
\hline & Control & 34.76 & 24.15 & \\
\hline
\end{tabular}

Table (4):-The mean values of different Salivary TGF- $\beta 1$ in both SSc patients and control subjects.

\begin{tabular}{|l|l|l|l|l|}
\hline & Groups & Median & Mean Rank & P value \\
\hline Saliva TGF- $\square$ lpg/ml & patients & 41.98 & 55.70 & $<0.001$ \\
\hline
\end{tabular}




\begin{tabular}{|l|l|l|l|l|}
\hline & Control & 22.61 & 25.30 & \\
\hline
\end{tabular}

\section{Discussion}

Serum and salivary IL-6:

The presentstudyshowed high significant levels of IL6 in all the biological samples tested (serum, saliva) ofSSc patients higher than that in the control subjects.

IL-6 is a cytokine with many biological effects on immune regulation, haematopoiesis, inflammation, oncogenesis, and it has been implicated in SSc pathogenesis. Previous studiesestablished thehigher production of IL6 by fibroblasts leading to increase the production of "collagen and glycosaminoglycans, hyaluronic acid and chondroitin-4/6-sulphates" from human dermal fibroblasts induced by IL6 in SSc patients [16].Serum Interlukines-6 increased in patients with SSc. This agree withprevious study [17].

Stimulation of fibroblasts by interleukin-6 (IL-6) is concerned in the pathogenesis of systemic sclerosis, proposing that the prevention of fibroblast activation may be anencouragingsystemic sclerosis treatment [18].

When SScfibroblasts are isolated and cultured in vitro, which stay to produce an additional of collagen. IL-6 is a profibrogenic cytokine, which stimulated the either "increase or decrease fibroblast proliferation, increase fibroblast collagen, glycosaminoglycan, and tissue inhibitor of metalloproteinases-1 (TIMP-1) synthesis, and increase MCP-1". IL-6controls the expression of "vascular endothelial growth factor (VEGF)", an important mediator ofangiogenesis and fibrosis, which is raised in patients withSSc[19].IL-6 has also been notice in "Sjogren's syndrome", and is known to be raisedin the saliva of Sjogren's syndrome patients. IL-6 have been also increased concentrations in the tear fluid of Sjogren's syndrome patients, with a significant positive correlation between IL-6 levels in tears and labial salivary gland (LSG) biopsy focus scores,production of cytokines by epithelial cells [20].The elevated concentration of IL-6 in the tear fluid of Sjogren's syndrome patients appears to reflect the production of this cytokine by conjunctival epithelial cells, as well as by inflammatory cells. Sections from LSGs were micro dissected to isolate infiltrating mononuclear cells, ductal cells, and acinar cells for separate analyses by RT-PCR. The study proves two conclusions; First, it was find that mRNA for many cytokines, including IL-2, IL-4, IL-6, IL-10, TNF-ot, IFN--y, and TGF13, could be find in all three types of samples. This finding firmly establishes that within the salivary glands, the epithelial cells themselves can influence the overall cytokine profile significantly.

Second, the researchers performed separate analyses of cytokine production by ductal and acinar epithelial cells that were adjacent or not adjacent to mononuclear cell foci [21].

\section{Serum and salivary TGF-B1:}

Serum TGF- $\beta$ was increased in patients with SSc. This comes into agreement with [22,23].

Multiple lines of evidence implicate TGF- $\beta$ as the master regulator of both physiologic fibrogenesis and pathological fibrosis Transforming growth factor $-\beta$ is the pre-eminent cue for initiating connective tissue remodeling during both normal wound healing and pathological fibrosis. The expression of receptors for TGF- $\beta$ as well as surface integrins that activate latent TGF- $\beta$ are elevated on SSc fibroblasts, suggesting that the SSc phenotype reflects autocrine TGF- $\beta$ stimulation of sensitized cells. Over three decades ago, CarwileLeRoy demonstrated that lesional skin fibroblasts explanted from patients with SSc synthesize increased amounts of collagen in vitro compared to healthy control fibroblasts. Additional features that phenotypically define SSc fibroblasts include increased synthesis of extracellular matrix; constitutive production of cytokines and chemokines; altered expression of cell surface integrins and receptors for transforming growth factor- $\beta$ (TGF- $\beta$ ), platelet-derived growth factor (PDGF) and the CC chemokine MCP-1; and my of ibroblasttrans differentiation[24].

TGF- $\beta 1$ is essential for wound healing, stimulates matrix molecule deposition, and is implicated in the pathogenesis of fibrotic disorders including SSc. Because prior studies demonstrated that Sema-7a promotes TGF- $\beta 1$ induced injury and fibrosis, and that TGF- $\beta 1$ induces the accumulation of fibrocytes[15].

\section{Bibliography\& References}

[1]. Ismet H.B., Kryeziu A, Sherifi F.Oral manifestations of Systemic Sclerosis and Correlation with anti-Topoisomerase I Antibodies (SCL-70)Med Arh. 2015 Jun; 69(3): 153-156

[2]. Mayes M.D., Lacey J.V., Beebe-Dimmer J, Gillespie B.W., Cooper B, Laing T.J., SchottenfeldD. Prevalence, incidence, survival, and disease characteristics of systemic sclerosis in a large US population. Arthritis Rheum 2003; 48:(8) 2246-55.

[3]. LeRoy E.C., Medsger T.A. Criteria for the classification of early systemic sclerosis. J Rheumatol 2001; 28(7):73-6.

[4]. Minier T, Guiducci S, Bellando-Randone S, et al. Preliminary analysis of the Very Early Diagnosis of Systemic Sclerosis EUST AR multicentre study: evidence for puffy finger s as a pivotal sign for suspicion of systemic sclerosis. Ann Rheum Dis. 2014; 73(12):2087-93.

[5]. Katsumoto T.R., Whitfield M.L., Connolly M.K. The pathogenesis of systemic sclerosis. Annu Rev Pathol 2011; 6:509-537. 
[6]. Knight V, Patricia A. Merkel and Michael D. O’Sullivan. Anticytokine Autoantibodies: Association with Infection and Immune Dysregulation Antibodies 2016, 5, 3

[7]. Scala E, Pallotta S, Frezzolini A, Abeni D, Barbieri C, Sampogna F, De Pità O, Puddu P, Paganelli R, Russo G. Cytokine and chemokine levels in systemic sclerosis: relationship with cutaneous and internal organ involvement. ClinExpImmunol 2004,138(3):540-546.

[8]. De Santis M, Bosello S, La Torre G, Capuano A, Tolusso B, Pagliari G, Pistelli R, Danza FM, Zoli A, Ferraccioli F. Functional, radiological and biological markers of alveolitis and infections of the lower respiratory tract in patients with systemic sclerosis. Respir Res 2005; 6:96-106

[9]. Tanaka T, Narazaki M, Ogata A, Kishimoto T. A new era for the treatment of inflammatory autoimmune diseases by interleukin-6 blockade strategy. Semin. Immunol. 2014, 26, 88-96.

[10]. Kadono T, Kikuchi K, Ihn H, Takehara K, Tamaki K. Increased production of interleukin 6 and interleukin 8 in scleroderma fibroblasts. J Rheumatol 1998; 25(2): 296-301.

[11]. Kondo K, Okada T, Matsui T, Kato S, Date K, Yoshihara M, Nagata Y, Takagi H, Yoneda M, Sugie I. Establishment and characterization of a human B cell line from the lung tissue of a patient with scleroderma; extraordinary high level of IL-6 secretion by stimulated fibroblasts. Cytokine 2001; 13(4):220-226.

[12]. Wu M.Y. and Hill C.S. TGF- $\beta$ superfamily signaling in embryonic development and homeostasis. Dev Cell 2009, 16:329-343.

[13]. Rosenbloom J, Castro S.V., Jimenez S.A. Narrative review: fibrotic diseases: cellular and molecular mechanisms and novel therapies. Ann Intern Med 2010; 152(3):159-166.

[14]. Gan Y, Reilkoff R, Peng X,Role of Semaphorin 7a signaling in TGF- $\beta 1$ induced lung fibrosis and scleroderma-related interstitial lung disease. Arthritis Rheum. 2011; 63(8): 2484-2494.

[15]. NavazeshM."Method for collecting saliva" Annals of the New York Academy of Science 1993; vol. 694,pp. 72-77

[16]. Scala E, Pallotta S, Frezzolini A, Abeni D, Barbieri C, Sampogna F, De Pità O, Puddu P, Paganelli R, Russo G. Cytokine and chemokine levels in systemic sclerosis: relationship with cutaneous and internal organ involvement. ClinExpImmunol 2004,138(3):540-546.

[17]. Yousifa M, Habib R, Esaely H, Yasin R, Sonbol A Interleukin-6 in systemic sclerosis and potential correlation with pulmonary involvement Egyptian Journal of Chest Diseases and Tuberculosis 2015; 64, 237-241

[18]. Tanaka T, Naka T, Kishimoto T, and Katayama I. Blockade of Interleukin-6 Receptor Alleviates Disease in Mouse Model of Scleroderma .The American Journal of Pathology, 2012; Vol. 180, No. 1.

[19]. Barnes T., Marina E. A., and Robert J. M. The Many Faces of Interleukin-6: The Role of IL-6 in Inflammation, Vasculopathy, and Fibrosis in Systemic Sclerosis International Journal of Rheumatology Volume 2011, 6page.

[20]. BishtR, and Sharma D. Sjogren'sdiseasa: A comprehansive review on recent clinical and experimental findings in pathogenesis and pharmacotherapyAsian Journal of Pharmaceutical and Clinical Research 2011; 1(4).

[21]. Ramos-Casals M, Tzioufas A.G., Stone J.H., Sisó A, Bosch X. Treatment of primary Sjögren syndrome: a systematic review. JAMA. 2010;304(4):452-60.

[22]. Uziel Y, Feldman B, Bernice R Krafchik, Ronald M Laxer and Rae SM Yeung. Increased serum levels of TGF 31 in children with localized Scleroderma Pediatric Rheumatology2007; 5:22, 1-5p

[23]. GeunC, Erica L. Herzog, ChitinaseF.A. Is a Biomarker for and Therapeutic Target in Scleroderma-Associated Interstitial Lung Disease That Augments TGF- $\beta 1$ Signaling J Immunol. 2012 September 1; 189(5): 2635-2644.

[24]. Wei J, Bhattacharyya S, Tourtellotte W.G., Varga J. Fibrosis in systemic sclerosis: Emerging concepts and implications for targeted therapy. Autoimmun Rev. 2011; 10(5): 267-275. 